\title{
Temperature Field-Wind Velocity Field Optimum Control of Greenhouse Environment Based on CFD Model
}

\author{
Yongbo Li, ${ }^{1}$ Guoxiang Sun, ${ }^{2}$ and Xiaochan Wang ${ }^{2}$ \\ ${ }^{1}$ College of Engineering, Nanjing Agricultural University, Nanjing 210031, China \\ 2 Jiangsu Province Engineering Lab for Modern Facility Agriculture Technology \& Equipment, Nanjing 210031, China
}

Correspondence should be addressed to Yongbo Li; bewit@njau.edu.cn

Received 27 March 2014; Revised 11 October 2014; Accepted 16 October 2014; Published 6 November 2014

Academic Editor: Valder Steffen Jr.

Copyright (c) 2014 Yongbo Li et al. This is an open access article distributed under the Creative Commons Attribution License, which permits unrestricted use, distribution, and reproduction in any medium, provided the original work is properly cited.

The computational fluid dynamics technology is applied as the environmental control model, which can include the greenhouse space. Basic environmental factors are set to be the control objects, the field information is achieved via the division of layers by height, and numerical characteristics of each layer are used to describe the field information. Under the natural ventilation condition, real-time requirements, energy consumption, and distribution difference are selected as index functions. The optimization algorithm of adaptive simulated annealing is used to obtain optimal control outputs. A comparison with full-open ventilation shows that the whole index can be reduced at $44.21 \%$ and found that a certain mutual exclusiveness exists between the temperature and velocity field in the optimal course. All the results indicate that the application of CFD model has great advantages to improve the control accuracy of greenhouse.

\section{Introduction}

In the greenhouse practice, the difference of growth and quality for the crop is an ordinary phenomenon, which could lead to a reduction of overall crop production [1]. A simulation based on Tomgro model also clearly shows that crops generally display the fast growth rate, high yield, and excellent quality in the central area; whereas crops in the peripheral area have the relatively slow growth rate, low yield, and worsening quality [2]. This unequilibrium has a lot of reasons, but the control system must be involved. Because it is generally assumed that the internal air in greenhouse is thoroughly mixed and homogeneous during the design of control system [3]. Based on this assumption, all models are applied in the design process.

In the early stage, the design of one-factor control system is generally based on the transfer function model [4]; and later, the design of the multifactor control system is developed in terms of the state space model [5]. Recently, the intelligent model has been more commonly applied [6]. Although these models are different, their designs are consistently based on the aforementioned assumptions. As a result, during the closed-loop feedback process with environmental factors, the "point" form is commonly used (sampling via a single sensor), and thus one feedback value is used to represent environmental conditions of the entire greenhouse space. However crops in the greenhouse are spatially distributed, and environmental factors at different locations could be manifested by different numerical values, the point forms to describe crops' requirements are inaccurate. So the field forms should be chosen to fit the real situations; in order to further improve the control precision of greenhouse environment, the selection of control model to explicitly reflect the "field" form is badly needed.

In recent years, the CFD technology has been fully developed and applied in the field of greenhouse environment modeling. The earliest exploration of the applicability and effectiveness of CFD technology for the simulation of greenhouse environment was studied by the literature [7]. During this period, greenhouse models were generally used in two-dimensional forms due to limitations of the computer hardware and software $[8,9]$. Later, with the dramatic increase of computational speed and improvement of algorithm efficiency, the greenhouse CFD model was extended to three-dimensional form [10]. 
Meanwhile, a variety of scenarios, such as greenhouse structures [11-13], geographical features [14, 15], environmental factors [16-18], and the existence/absence of crops $[19,20]$, were included in the construction of greenhouse CFD model, and the results generally displayed that utilizing CFD technology to establish greenhouse environment model is effective. In theory, CFD model is based on basic principles such as the conservation of energy, mass, and momentum. During the establishment of the model, the nonlinearity and coupling features of greenhouse have been fully taken into account; hence, the description precision of the model is high. Another advantage lies in the fact that, through mesh generation, the factor state of any points within the greenhouse space could be expressed theoretically. Therefore, the integration of CFD in greenhouse environment model could meet the requirement of "field" form, which could help planters to control the overall greenhouse state, not to limit it to one point for part of crops.

Currently, the use of CFD as the greenhouse environment model is commonly applied for the short-term forecasting and indirect guidance of environment control $[11,13,14,16]$. In the literature [21-25], the design of experiment (DOE) and genetic algorithm (GA) to control greenhouse temperature field under CFD model were applied. However, these studies are merely limited to a single factor, which could not provide a complete description of the state of greenhouse environment. Hence, this paper selects two factors (temperature and wind velocity) as the greenhouse control object under the CFD greenhouse model. Different from the common design, the wind velocity was chosen as a control variable. The reason is that the wind velocity is a very important factor if we want to control the distributions of humidity and $\mathrm{CO}_{2}$ concentration. This point has been testified by our experiments and former research literatures. Therefore, the control for the distributions of humidity or $\mathrm{CO}_{2}$ concentration can be changed accordingly to control the wind velocity.

The paper's structure is as follows. Firstly, the natural ventilation is chosen as the control pattern to set up the whole system. The field information which includes the maximum value, average value, minimum value, and standard deviation is achieved by digitizing the height of greenhouse space. Secondly, with a careful consideration, a multiple index system for greenhouse control is obtained, which contained the energy confinement, real-time control, temperature, wind velocity field, and so forth. Finally, adaptive simulated annealing (ASA) algorithm is applied as the optimization algorithm to solve the optimal control problem and get the natural ventilation strategy of using the CFD greenhouse model for the temperature field-wind velocity field (TF-WVF) under the condition of multiconstraints and multitargets.

\section{Greenhouse Model}

2.1. Physical Model. The greenhouse is located in Nanjing Agricultural University (E $\left.118^{\circ} 46^{\prime}, \mathrm{E} 32^{\circ} 03^{\prime}\right)$. It is 2-ridge Venlo structure with aluminium alloy material, and size is $8 \mathrm{~m}$ (width) $\times 16 \mathrm{~m}$ (length); gutter and ridge height are $4.2 \mathrm{~m}$ and $5.2 \mathrm{~m}$, respectively; the total area is $128 \mathrm{~m}^{2}$. The greenhouse

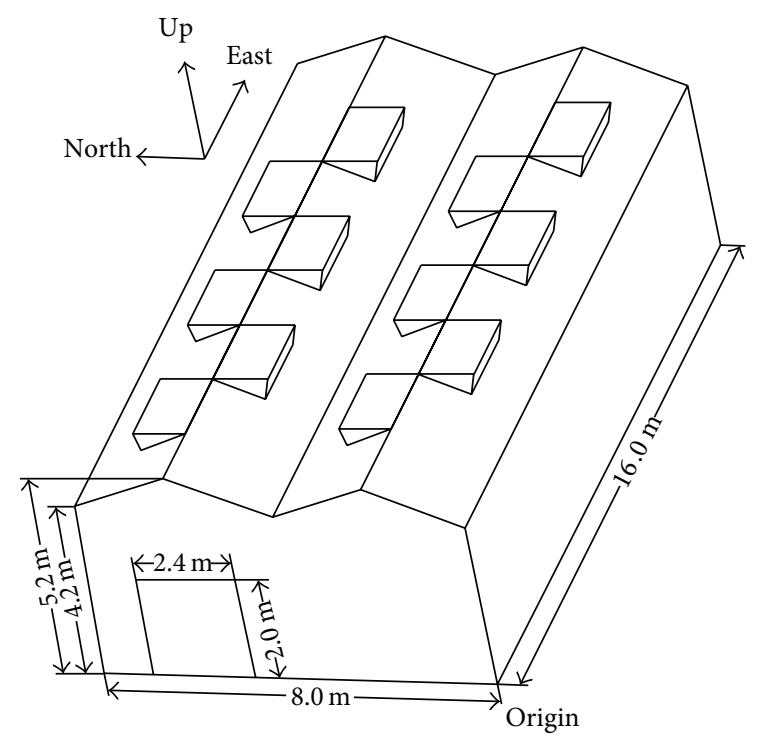

FIGURE 1: Greenhouse physics structure.

is covered with $4 \mathrm{~mm}$ float glass whose light transmission is over $88 \%$. Environment control devices include window opening, external/internal sun shading, spraying, and drip irrigation. The natural ventilation consists of 6 windows that open to the north and 6 that open in the opposite direction and an inlet which is located in west front; all of windows have a synchronous moving mode. Geometric structure of greenhouse is shown in Figure 1.

2.2. Greenhouse CFD Model. For setting up the CFD model in Figure 1, continuity equation, momentum equation, energy equation, three-dimensional turbulence equation, radiation equation, and solar load model are embedded in greenhouse model. During the CFD calculation process, it is assumed that the temperature is a well-distribution even through glass layers of the outer covering; translucent materials are used for the construction of the roof and sides area; the concrete structure is used for the ground; the absorption rate of radiation energy remains constant; and the heat is transferred via the form of fixed absorption [21].

2.3. Description of Calculation Software and Hardware. The mesh and fluid calculations were made by using the software: Gambit 2.4.6 and Fluent 6.3.26, respectively. One velocity inlet, twelve skylight pressure outlets, five glass walls, and one concrete wall (ground) are set up. The computer mode is Lenovo Ideapad Z370, with CPU as Intel (R) Celeron (TM) I3-2350M $4 \times 2.30 \mathrm{GHz}$, memory as DDR3 $1333 \mathrm{MHz} 4 \mathrm{~GB}$, and hard disk as ST500LM012 with the total of $500 \mathrm{G}$ storage. The operating system is 32-bit ultimate version of Windows 7 , and four-thread parallel computing is adopted during the process of Fluent iterative calculation. In the experiments, the initial conditions and boundary conditions in Fluent and Gambit were set as Table 1. 
TABLE 1: Initial conditions and boundary conditions.

\begin{tabular}{lc}
\hline Parameters name (unit) & Value \\
\hline Air & 1.225 \\
Density $\left(\mathrm{kg} \cdot \mathrm{m}^{-3}\right)$ & 0.0242 \\
Heat coefficient $\left(\mathrm{W} \cdot(\mathrm{m} \cdot \mathrm{K})^{-1}\right)$ & $3.356 e-3$ \\
Thermal expansion coefficient $\left(\mathrm{K}^{-1}\right)$ & 1006.43 \\
$\quad$ Specific heat capacity $\left(\mathrm{kJ} \cdot(\mathrm{kg} \cdot \mathrm{K})^{-1}\right)$ & \\
Glass & 2220 \\
Density $\left(\mathrm{kg} \cdot \mathrm{m}^{-3}\right)$ & 1.15 \\
Heat coefficient $\left(\mathrm{W} \cdot(\mathrm{m} \cdot \mathrm{K})^{-1}\right)$ & 830 \\
Specific heat capacity $\left(\mathrm{kJ} \cdot(\mathrm{kg} \cdot \mathrm{K})^{-1}\right)$ & 0.004 \\
Thickness $(\mathrm{m})$ & \\
Boundary conditions & 2.0 \\
Inlet velocity $\left(\mathrm{m} \cdot \mathrm{s}^{-1}\right)$ & 300 \\
Inlet temperature $(\mathrm{K})$ & 305 \\
Outdoor temperature $(\mathrm{K})$ & 300 \\
Glass wall temperature $(\mathrm{K})$ & 290 \\
$\quad$ Ground wall temperature $(\mathrm{K})$ &
\end{tabular}

\section{Control Objects in Field Form}

Under the "point" feedback form, the control objective is a constant, whereas under the "field" feedback form, the control object needs to be expanded that it should thoroughly reflect the spatial distribution of greenhouse environmental factors. So we will decide the control object from two aspects: constraint and index.

\subsection{Control Constraints}

3.1.1. Time Constraint of CFD Computing. Since the CFD model is a nonlinear system, its calculation process is timeconsuming. In order to enhance the feasibility in practice, constraints should contain model parameters, the selection of equations, the choice of appropriate algorithms, the determination of the convergence level, and so forth. Here the calculation time of CFD model should meet the requirements of (1) as follows:

$$
T_{\mathrm{CFD}}<T_{s}
$$

where $T_{\text {CFD }}$ represents the required computing time of CFD model during the optimization process and $T_{s}$ represents the sampling period of the control system. Additionally, (1) requires sufficient calculation effectiveness of CFD model in Section 2.2 so that the manipulation time under the realistic setting could be met, and the optimization requirement of control algorithm for subsequent steps could be provided.

3.1.2. Constraint of TF-WVF. In order to achieve the extended form of "point" feedback to "field" form, this paper uses height as the reference and chooses the extreme values (maximum and minimum value) and average value obtained from different horizontal planes as numerical characteristics (NC) to describe the TF-WVF information database based on CFD model. Expressions of TF and WVF are given as follows.

\section{(1) NC of TF:}

$$
\begin{aligned}
& \max T=\max \left\{T_{i}\right\}, \\
& \min T=\min \left\{T_{i}\right\}, \\
& \operatorname{ave} T=\text { ave }\left\{T_{i}\right\},
\end{aligned}
$$

where $\max T, \min T$, and ave $T$ represent the maximum temperature, minimum temperature, and average temperature, respectively; these values correspond to the height of $h$ of the horizontal plane, $i$ represents the mesh point number after mesh generation, and $T_{i}$ represents the temperature feedback value of point $i$.

(2) NC of WVF:

$$
\begin{aligned}
& \max V=\max \left\{\left|\vec{V}_{i}\right|\right\}, \\
& \min V=\min \left\{\left|\vec{V}_{i}\right|\right\}, \\
& \operatorname{ave} V=\text { ave }\left\{\left|\vec{V}_{i}\right|\right\},
\end{aligned}
$$

where $\max V, \min V$, and aveV represent the maximum velocity, minimum velocity, and average velocity, respectively, which corresponds to the horizontal plane with a height of $h$ and $\vec{V}_{i}$ represents the wind velocity vector of point $i$ after mesh generation of that plane.

It can be seen that, different from the temperature field, the wind velocity field is in a vector format, and thus its amplitude value is calculated in accordance with the following:

$$
\left|\vec{V}_{i}\right|=\sqrt{\left(V_{i}^{x}\right)^{2}+\left(V_{i}^{y}\right)^{2}+\left(V_{i}^{z}\right)^{2}},
$$

where $V_{i}^{x}, V_{i}^{y}$, and $V_{i}^{z}$ represent the velocity components of point $i$ along three directions.

Although the range of height $h \in\left[\begin{array}{ll}0 & y_{\max }\end{array}\right]$, which is continuous $\left(y_{\max }\right.$ represents the maximum height of the greenhouse), growers' major concern is about some key heights in practice, such as ground layer, intermediate layer, leaf layer, and canopy layer. Considering our experimental object is the tomato in greenhouse. In this paper, we divided the horizontal planes into six different levels including $0.2 \mathrm{~m}$, $0.4 \mathrm{~m}, 0.8 \mathrm{~m}, 1.0 \mathrm{~m}, 1.2 \mathrm{~m}$, and $2.0 \mathrm{~m}$.

3.2. Control Indexes Set. During the process of greenhouse production, many aspects should be involved in control system. This paper explicitly considers three aspects, including the control cost index, distribution difference index, and overall index; the details of indexes are given as follows.

3.2.1. Control Cost Index. The control cost index refers to the amount of energy consumption of related equipment under natural ventilation conditions. In this paper, both the greenhouse entrance and the skylight are controlled by motors which are the same mode. Therefore, the control cost index could be described as follows:

$$
J_{u}=\sum_{k=1}^{3} \lambda_{k} u_{k},
$$


where $J_{u}$ represents the overall cost index. $k=1,2,3$ represents the control output of entrance, north skylight, and south skylight, respectively. $\lambda_{k}$ represents respective proportion of motor power with $\sum_{k=1}^{3} \lambda_{k}=1$. The considered three kinds of motors belong to the same mode with $\lambda_{1}=\lambda_{2}=$ $\lambda_{3}=1 / 3 . u_{k} \in\left(\begin{array}{ll}0 & 100 \%\end{array}\right)$ represents respective openness.

Note. The experimental greenhouse is small and the cost of electric motor is less. However, in the practical greenhouse, especially greenhouse-group, the cost of running electric motor is a very important factor for planters' income.

3.2.2. Distribution Difference Index. The distribution difference index refers to the difference in the distribution of environmental factors under a certain level of height $h$; smaller index values indicate that the wind velocity or temperature is evenly distributed at the plane; thus the variation in the growth status of crops could be reduced. Therefore, if the control system can regulate the devices to decrease this index which satisfied the demand of all crops in greenhouse, the greenhouse yield will be enlarged and the precision of control system will be dramatically enhanced. This paper uses the mean standard deviation to describe the distribution difference level of TF-WVF.

(1) TF distribution difference:

$$
J_{T}=\sqrt{\frac{1}{N} \sum_{i=1}^{N}\left(T_{i}-\operatorname{ave} T\right)^{2}},
$$

where $J_{T}$ represents the distribution difference of temperature and $N$ represents the number of nodes at the height of $h$ after being meshed by Gambit.

Considering layers are limited, stratified (6) can get

$$
J_{T}=\sum_{m=1}^{6} a_{m} J_{T}^{m},
$$

where $a_{m}$ represents the weight coefficient at layer $m$ with $\sum_{m=1}^{6} a_{m}=1$ and $J_{T}^{m}$ represents the distribution difference index at layer $m$.

(2) WVF distribution difference:

$$
J_{V}=\sqrt{\frac{1}{N} \sum_{i=1}^{N}\left(\left|\vec{V}_{i}\right|-\operatorname{ave} V\right)^{2}}
$$

where $J_{V}$ represents WVF distribution difference and the calculation of $\left|\vec{V}_{i}\right|$ is referred to (4). After similar stratification and description process, we have

$$
J_{V}=\sum_{m=1}^{6} b_{m} J_{V}^{m}
$$

where $b_{m}$ represents the weight coefficient of layer $m$ with $\sum_{m=1}^{6} b_{m}=1$ and $J_{V}^{m}$ represents distribution difference index at layer $m$.
3.2.3. Overall Index. In this paper, two involved environmental factors are the wind velocity and temperature. Meanwhile, the control indexes include real-time, cost, and distribution difference. Therefore, the control system is multiconstraint and multi-index. In order to take all these characteristics into account, the following overall index is proposed:

$$
J=\alpha J_{T}+\beta J_{V}+\gamma J_{u},
$$

where $\alpha, \beta$, and $\gamma$ are weight coefficient of three indexes. Here, we set $\alpha=\beta=0.4$ and $\gamma=0.2$.

\section{The Optimal Control}

Constraints and indexes obtained under field form conditions; the natural ventilation optimization control system based on CFD model could be described as shown in Figure 2.

Figure 2 displays a closed-loop control system. The CFD model and the control system are linked via the extraction of field information which is based on Section 3. The optimization control system applies ASA algorithm to solve the following optimization problem with a wide stochastic optimal course as follows:

$$
\begin{array}{ll}
\min & J=\min \left(\alpha J_{T}+\beta J_{V}+\gamma J_{u}\right) \\
\text { s.t. } & T_{\mathrm{CFD}}<T_{S} \\
& \text { ave } T \in \Omega_{T} \\
& \text { ave } V \in \Omega_{V},
\end{array}
$$

where $\Omega_{T}=(295 \pm 2) \mathrm{K}$ represents the average temperature requirement of the corresponding layers and $\Omega_{V} \epsilon$ $(0.3 \pm 0.1) \mathrm{m} / \mathrm{s}$ represents the average wind velocity. All the parameters set in this section are only to testify the feasibility of the CFD technology.

\section{Results and Discussions}

The natural ventilation is an ordinary regulation method for the greenhouse control, and in practice, it is often in full-action (full open or close) or inaccurate controlled by the planter. So in this paper, the continuous and accurate action mode will be studied. To make a comparison, the field information and related indexes under full open natural ventilation (FONV) conditions are researched firstly. Next, under the same indexes the optimal control system will be analyzed completely. By comparison and analysis, the effective information would be collected for the guidance of system design in practice.

5.1. FONV. The meaning of FONV is $u_{1}=u_{2}=u_{3}=100 \%$. Under this condition, the previous mentioned greenhouse environment information which includes NCs of wind velocity-temperature factors and related indexes could be calculated. Some results are given in Table 2.

Table 2 represents the numerical characteristics and related indexes of the six layers. It can be seen from this 


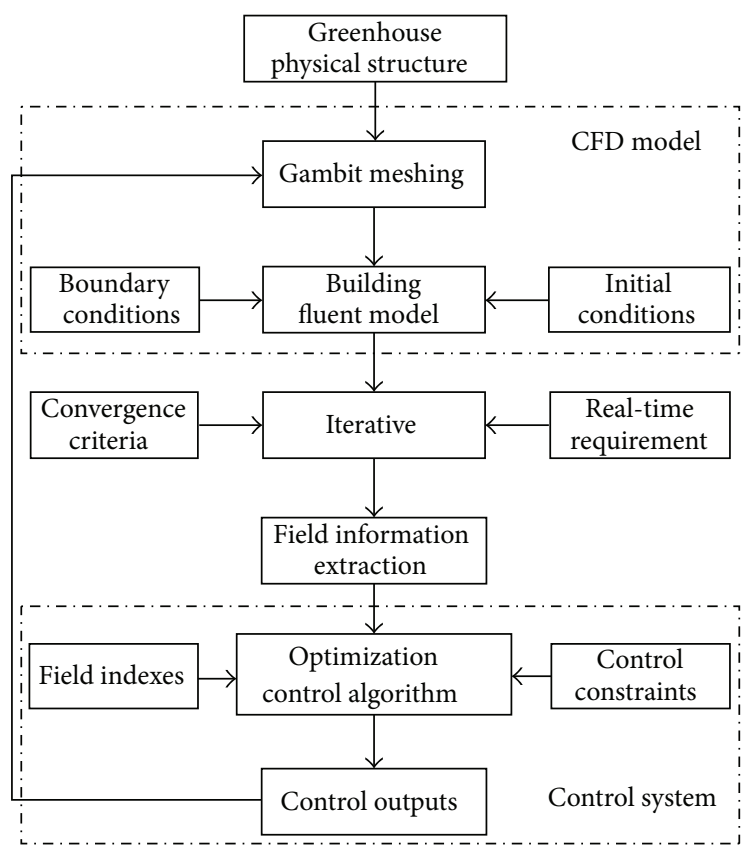

FIgURe 2: Principle of control system.

TABLE 2: NCs of FONV.

\begin{tabular}{|c|c|c|c|c|c|c|}
\hline$h(\mathrm{~m})$ & 0.2 & 0.4 & 0.8 & 1.0 & 1.2 & 2.0 \\
\hline \multicolumn{7}{|l|}{$T(\mathrm{~K})$} \\
\hline Min & 294.35 & 294.43 & 294.54 & 294.59 & 294.64 & 294.70 \\
\hline Ave & 294.75 & 294.89 & 294.94 & 294.96 & 294.98 & 295.01 \\
\hline Max & 296.20 & 295.95 & 295.73 & 295.71 & 295.67 & 295.80 \\
\hline \multicolumn{7}{|l|}{$V(\mathrm{~m} / \mathrm{s})$} \\
\hline Min & 0.014299 & 0.018991 & 0.031150 & 0.032261 & 0.036340 & 0.056740 \\
\hline Ave & 0.832410 & 0.817559 & 0.803519 & 0.799759 & 0.796762 & 0.756358 \\
\hline Max & 3.042729 & 3.131846 & 3.047643 & 3.035799 & 3.035799 & 3.017646 \\
\hline SDT (K) & 0.18608 & 0.15697 & 0.13566 & 0.12649 & 0.12165 & 0.11381 \\
\hline $\operatorname{SDV}(\mathrm{m} / \mathrm{s})$ & 0.76187 & 0.75538 & 0.74742 & 0.74337 & 0.74227 & 0.71905 \\
\hline
\end{tabular}

table that for either temperature or wind velocity, the corresponding average value closely relates with the height. That is to say, the average value of temperature increases with the increasing height, whereas the average value of wind velocity decreases with the increasing height, indicating that among greenhouse environmental factors, the temperature and wind velocity are negatively correlated. Meanwhile, there is a negative correlation between the temperature and wind velocity distribution too. From Table 2, it is clear that $J_{T}=$ $0.1357, J_{V}=0.7461$, and $J_{u}=1.0$. For Table 2 , the overall index under the optimal scenario is 0.5528 . All of these results under full ventilation conditions will be used as a reference to compare.

For a visual expression, we choose the middle layer (height is $1.0 \mathrm{~m}$ ) to display the cloud map which contains the temperature and wind velocity in Figure 3.

Figure 3(a) shows the temperature cloud map with $h=$ $1.0 \mathrm{~m}$. It can be seen from this figure that, for most part of the layer, the average temperature is around $295 \mathrm{~K}$ with some local variation. Figures 3(b) and 3(c) show the wind velocity contours along $x$ and $z$ directions, respectively. Clearly, the velocity along $x$ direction is slower than that along the $z$ direction; the reason could be explained by the direction of the greenhouse entrance. Meanwhile, two contours display significant turbulence phenomena, which are quite similar to Figure 3(a), indicating that temperature and wind velocity factors are coupled strongly.

5.2. Optimal Scenario. In this pattern, the optimization processes spend about 61 minutes to attain the control outputs $\left(u_{1}=22.62 \%, u_{2}=93.59 \%\right.$, and $\left.u_{3}=10.95 \%\right)$. The temperature-wind velocity cloud map at height of $h=1.0 \mathrm{~m}$ is shown in Figure 4, and the NCs of the six layers are shown in Table 3.

It can be seen from the cloud map that the isotherms include $294.5 \mathrm{~K}, 295 \mathrm{~K}$, and $295.5 \mathrm{~K}$. Although the distribution is similarly compared to FONV, the distribution difference is reduced, and the double-eddy phenomenon 


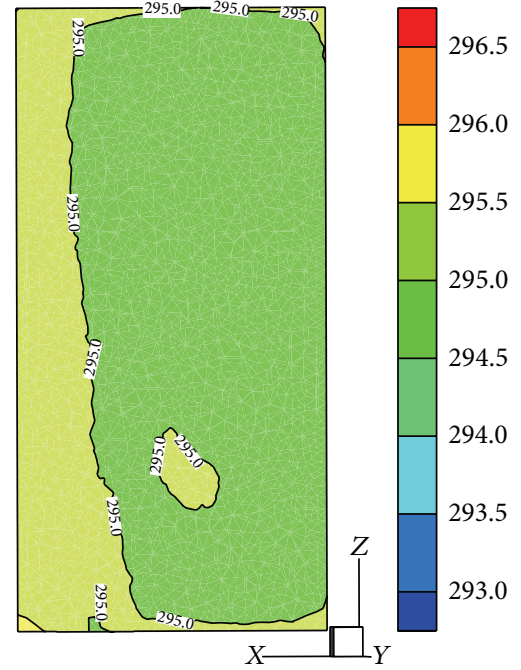

(a) Temperature

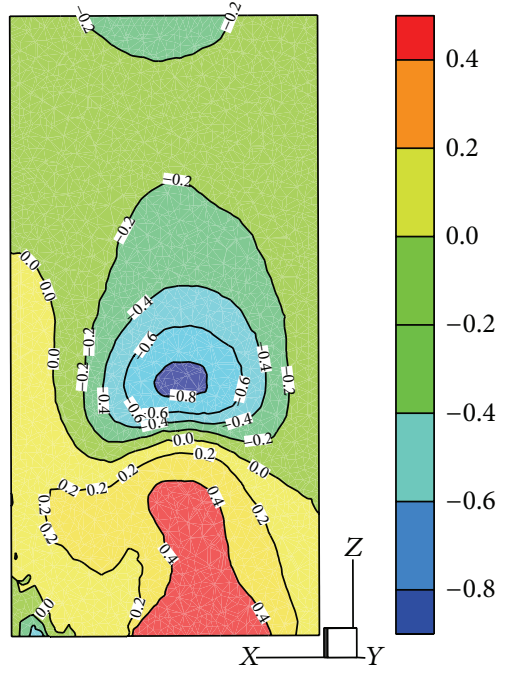

(b) Velocity of $x$ direction

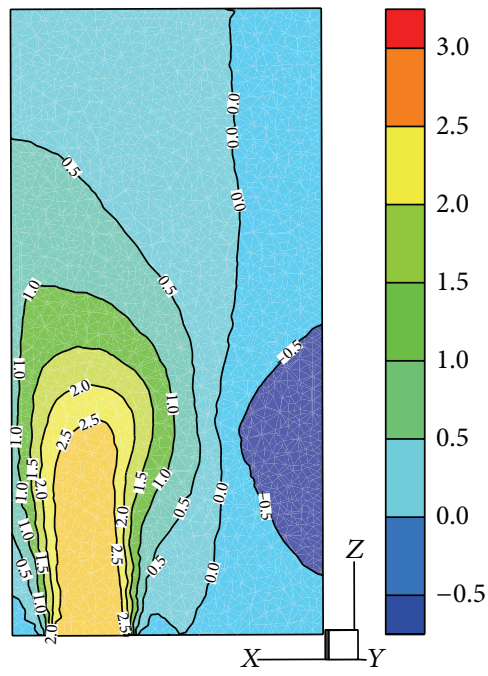

(c) Velocity of $z$ direction

Figure 3: Cloud map of FONV at $1.0 \mathrm{~m}$.

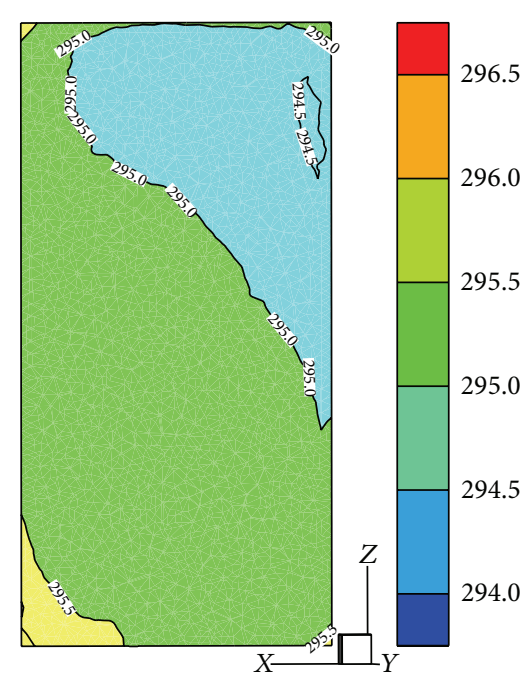

(a) Temperature

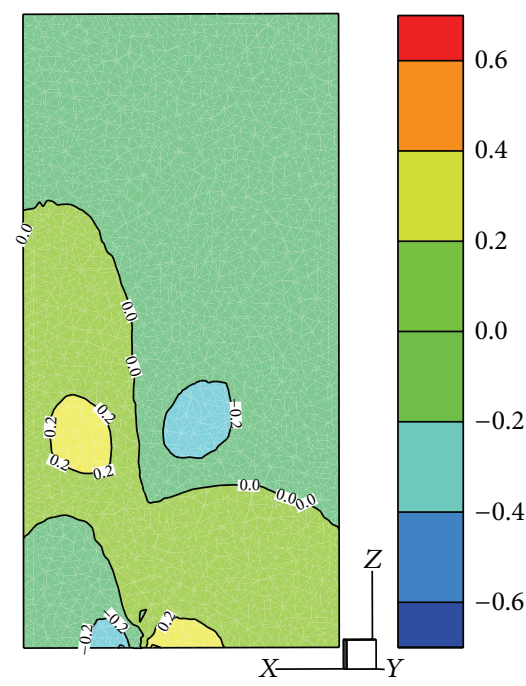

(b) Velocity of $x$ direction

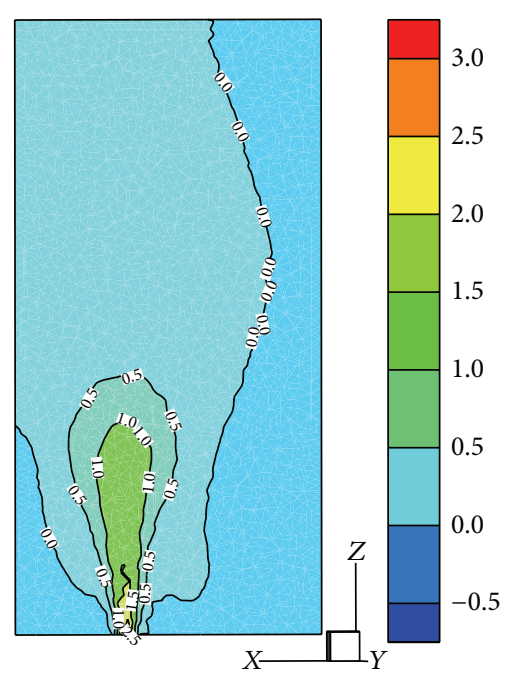

(c) Velocity of $z$ direction

FIgURE 4: Cloud map of scenario 1 at $1.0 \mathrm{~m}$.

TABLE 3: NCs of optimal scenario.

\begin{tabular}{|c|c|c|c|c|c|c|}
\hline$h(\mathrm{~m})$ & 0.2 & 0.4 & 0.8 & 1.0 & 1.2 & 2.0 \\
\hline \multicolumn{7}{|l|}{$T(\mathrm{~K})$} \\
\hline Min & 293.65 & 293.97 & 294.20 & 294.24 & 294.33 & 294.46 \\
\hline Ave & 294.88 & 295.02 & 295.09 & 295.11 & 295.13 & 295.17 \\
\hline Max & 296.33 & 296.53 & 296.21 & 296.13 & 296.09 & 296.52 \\
\hline \multicolumn{7}{|l|}{$V(\mathrm{~m} / \mathrm{s})$} \\
\hline Min & 0.001656 & 0.002452 & 0.007214 & 0.008335 & 0.010763 & 0.014362 \\
\hline Ave & 0.270686 & 0.254144 & 0.233365 & 0.224360 & 0.215042 & 0.157519 \\
\hline Max & 2.997552 & 3.063393 & 3.002424 & 2.955838 & 2.941845 & 3.117379 \\
\hline SDT (K) & 0.32906 & 0.28879 & 0.24432 & 0.22823 & 0.21794 & 0.21176 \\
\hline $\operatorname{SDV}(\mathrm{m} / \mathrm{s})$ & 0.33999 & 0.33691 & 0.32172 & 0.31735 & 0.30061 & 0.22776 \\
\hline
\end{tabular}


could be found in the wind field that the amplitude is reduced as well. NCs of six different layers are calculated, including the average temperature, the average velocity, SDT, and SDV. Table 3 has a similar regulation with Table 2 .

5.3. Comparing and Conclusions. To make a comparison, we choose (12) to define the difference between FONV and optimal scenario as follows:

$$
\text { Difference }=\text { Optimal }- \text { FONV }
$$

Note. The horizontal axis is about the height of the layer, and the vertical layer is the average temperature, the average velocity, SDT, or SDV, respectively.

Figure 5 shows that both the average temperature and SDT are positive. Therefore, during the optimization process, the average temperature and the temperature distribution are both positive at each layer; these results show that the indexes of temperature are not better than the FONV. In other words, full ventilation mode represents the best ventilation form to reduce distribution for temperature, but only in temperature. Meanwhile, the average velocity was decreased because the $u_{1}$ is less than $100 \%$; it led to the decrease of air exchange. The SDV is negative and indicates that the velocity distribution became better than FONV; these results can be explained by turbulent theory that the turbulence intensity has the same change direction with $u$. This phenomenon can also be conformed from an extreme case: when the greenhouse is closed the wind will not flow, so the aveV $\approx 0$ and SDV $\approx$ 0 ; this is the best way to reduce SDV but does not suit the greenhouse control. To further enrich the information differences between FONV and optimal scenario, we make Table 4 to describe it.

In the optimal scenario, the values of $\alpha=\beta=0.4$ and $\gamma=0.2$ indicate that this mode belongs to a comprehensive optimization system, which includes the temperature, velocity, and control cost. Therefore, this scenario has a wide variety of applications in practice. Except for $J_{T}$, values of all other indexes are reduced, with the overall index reduced $44.21 \%$, indicating that the optimization system in this paper has a great superiority for application in terms of greenhouse environment control.

\section{Conclusions}

By theoretical design and a specific calculation example, the following conclusions have been obtained.

(1) The optimization control method of greenhouse TFWVF based on the CFD model is effective, which could extend the conventional "point" feedback forms to "field" feedback forms. Therefore, the new method will be a good strategy to control the distribution of greenhouse environmental factors with multi-index constrained.

(2) The calculation example shows that the distribution of overall optimization index is reduced by $44.21 \%$. The optimal scenario indicates that it is advantageous to

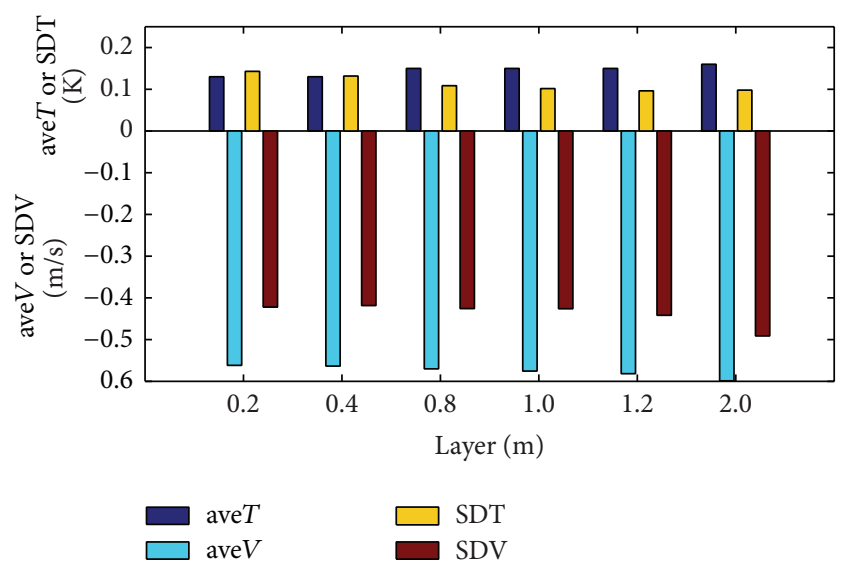

FIGURE 5: NCs difference between optimal scenario and FONV.

TABLE 4: Compared result of indexes with FONV.

\begin{tabular}{lccc}
\hline & \multirow{2}{*}{ FONV } & \multicolumn{2}{c}{ Optimal scenario } \\
& & Value & Variation \\
\hline$J_{T}$ & 0.1357 & 0.2457 & $+80.95 \%$ \\
$J_{V}$ & 0.7461 & 0.3154 & $-57.72 \%$ \\
$J_{u}$ & 1.0000 & 0.4196 & $-58.04 \%$ \\
\hline$J$ & 0.5528 & 0.3084 & $-44.21 \%$ \\
\hline
\end{tabular}

explore the potentials of greenhouse control system when the CFD model is applied.

(3) Among the NCs of six layers in the optimal scenario and FONV, the average temperature increases with the height, whereas the average velocity decreases with the height. Furthermore, both the temperature and velocity distributions are increased with the height; the result indicates that the height and distribution are negatively correlated.

(4) In comparison with the FONV, the change of absolute value is different, $\Delta J_{V}=0.431, \Delta J_{T}=0.110$, so we can conclude that the WVF optimal has a more potential to dig than TF.

A note must be explained. Although the single CFD cycle is set less than $T_{\mathrm{CFD}}$, the optimum course has a long time (61 minutes). In order to practice this way to control greenhouse environment, we must pay more attention to enhance the algorithm efficiency and adopt multicore parallel computing to reduce the time cost. If the optimal course can be limited in about 15 minutes, the CFD model for greenhouse control will be applied in practice. 


\section{Nomenclature}

CFD: Computational fluid dynamics

TF-WVF: Temperature field and wind velocity field

DOE: Design of experiment

GA: $\quad$ Genetic algorithm

ASA: $\quad$ Adaptive simulated annealing

NC: $\quad$ Numerical characteristic

FONV: Full open natural ventilation

SDT: $\quad$ Standard deviation of temperature

SDV: $\quad$ Standard deviation of velocity

max: $\quad$ The maximum value

min: The minimum value

ave: The average value

$T_{i}$ : $\quad$ Cell temperature

$\vec{V}_{i}$ : $\quad$ Vector of cell velocity

$\left|\vec{V}_{i}\right|: \quad$ Amplitude of cell velocity

J: $\quad$ Whole index

$J_{T}: \quad$ Temperature index

$J_{V}: \quad$ Velocity index

$J_{u}: \quad$ Energy index

$i$ : $\quad$ Cell point in a plane

$u$ : $\quad$ Control input

a: $\quad$ Temperature weight coefficient

$b$ : Velocity weight coefficients.

\section{Greek Symbols}

$\lambda_{k}$ : Weight coefficients of control input

$\alpha$ : Temperature weight coefficient

$\beta$ : Velocity weight coefficient

$\gamma$ : Energy cost weight coefficient.

\section{Superscript}

$x, y, z$ : Coordinate direction

$m$ : Height of layer

$\Omega_{T}: \quad$ Range of average temperature

$\Omega_{V}$ : Range of average wind velocity.

\section{Conflict of Interests}

The authors declare that there is no conflict of interests regarding the publication of this paper.

\section{Acknowledgments}

This work is supported by the National Natural Science Foundation of China (Grant no. 61273227) and Northern Jiangsu Science and Technology Development Plan (Grant no. BN2013051).

\section{References}

[1] J. Wang and B. Zhang, "Application of some cooling measures for greenhouse cooling in summer," Transactions of the Chinese
Society of Agricultural Engineering, vol. 22, no. 9, pp. 257-260, 2006.

[2] C. R. Bojacá, R. Gil, and A. Cooman, "Use of geostatistical and crop growth modelling to assess the variability of greenhouse tomato yield caused by spatial temperature variations," Computers and Electronics in Agriculture, vol. 65, no. 2, pp. 219-227, 2009.

[3] A. J. Udink ten Cate, Modeling and adaptive control of greenhouse climate [Ph.D. dissertation], Agricultural University, Wageningen, The Netherlands, 1983.

[4] A. Setiawan, L. D. Albright, and R. M. Phelan, "Application of pseudo-derivative-feedback algorithm in greenhouse air temperature control," Computers and Electronics in Agriculture, vol. 26, no. 3, pp. 283-302, 2000.

[5] N. Bennisa, J. Duplaixb, G. Eneab, M. Halouac, and H. Youlald, "Greenhouse climate modelling and robust control," Computers and Electronics in Agriculture, vol. 26, no. 9, pp. 96-107, 2008.

[6] F. Fourati and M. Chtourou, "A greenhouse control with feedforward and recurrent neural networks," Simulation Modelling Practice and Theory, vol. 15, no. 8, pp. 1016-1028, 2007.

[7] L. Okushima, S. Sase, and M. Nara, "A support system for natural ventilation design of greenhouses based on computational aerodynamics," Acta Horticulture, vol. 248, no. 1, pp. 129-136, 1989.

[8] R. Haxaire, J. C. Roy, T. Boulard et al., "Experimental and numerical study of a free-convective ventilation in a greenhouse," Colloque Annuel-SFT, vol. 109, no. 6, pp. 64-69, 1998.

[9] T. H. Short, "Aerodynamic design improves ventilation," Grower Talks Magazine, vol. 12, no. 7, pp. 90-98, 1998.

[10] K. Kim, J.-Y. Yoon, H.-J. Kwon et al., "3-D CFD analysis of relative humidity distribution in greenhouse with a fog cooling system and refrigerative dehumidifiers," Biosystems Engineering, vol. 100, no. 2, pp. 245-255, 2008.

[11] B. L. Gowreesunker and S. A. Tassou, "Effectiveness of CFD simulation for the performance prediction of phase change building boards in the thermal environment control of indoor spaces," Building and Environment, vol. 59, pp. 612-625, 2013.

[12] M. Abbes, A. Farhat, A. Mami, and G. Dauphin-Tanguy, "Pseudo bond graph model of coupled heat and mass transfers in a plastic tunnel greenhouse," Simulation Modelling Practice and Theory, vol. 18, no. 9, pp. 1327-1341, 2010.

[13] P. A. Davies and C. Paton, "The seawater greenhouse in the United Arab Emirates: thermal modelling and evaluation of design options," Desalination, vol. 173, no. 2, pp. 103-111, 2005.

[14] G. Tong, D. M. Christopher, and B. Li, "Numerical modelling of temperature variations in a Chinese solar greenhouse," Computers and Electronics in Agriculture, vol. 68, no. 1, pp. 129139, 2009.

[15] H. Majdoubi, T. Boulard, H. Fatnassi, and L. Bouirden, "Airflow and microclimate patterns in a one-hectare Canary type greenhouse: an experimental and CFD assisted study," Agricultural and Forest Meteorology, vol. 149, no. 6-7, pp. 1050-1062, 2009.

[16] A. López, D. L. Valera, F. D. Molina-Aiz, and A. Peña, "Sonic anemometry to evaluate airflow characteristics and temperature distribution in empty Mediterranean greenhouses equipped with pad-fan and fog systems," Biosystems Engineering, vol. 113, no. 4, pp. 334-350, 2012.

[17] R. Wang, H. Xu, and J. Ma, "CFD analysis of airflow distribution in greenhouse with pad and fan cooling system," Transactions of the CSAE, vol. 27, no. 6, pp. 250-255, 2011. 
[18] D. Piscia, J. I. Montero, E. Baeza, and B. J. Bailey, "A CFD greenhouse night-time condensation model," Biosystems Engineering, vol. 111, no. 2, pp. 141-154, 2012.

[19] R. Nebbali, J. C. Roy, and T. Boulard, "Dynamic simulation of the distributed radiative and convective climate within a cropped greenhouse," Renewable Energy, vol. 43, pp. 111-129, 2012.

[20] A. Kichah, P.-E. Bournet, C. Migeon, and T. Boulard, "Measurement and CFD simulation of microclimate characteristics and transpiration of an impatiens pot plant crop in a greenhouse," Biosystems Engineering, vol. 112, no. 1, pp. 22-34, 2012.

[21] Y. Li, W. Zhou, P. Li, X. Wang, and W. Ding, "Temperature homogeneity control of greenhouse based on CFD simulation model," Transactions of the Chinese Society of Agricultural Machinery, vol. 43, no. 4, pp. 156-161, 2012.

[22] L. Yongbo, S. Guoxiang, L. Enping, W. Xiaochan, and D. Weimin, "Multi-index GA optimal control of greenhouse temperature based on CFD model," Transactions of the Chinese Society for Agricultural Machinery, vol. 44, no. 3, pp. 186-191, 2013.

[23] A. Ousegui, C. Moresoli, M. Dostie, and B. Marcos, "Optimal control and CFD modeling for heat flux estimation of a baking process," Computers and Chemical Engineering, vol. 38, pp. 139150, 2012.

[24] H. Huang, R. Ooka, H. Chen, and S. Kato, "Optimum design for smoke-control system in buildings considering robustness using CFD and Genetic Algorithms," Building and Environment, vol. 44, no. 11, pp. 2218-2227, 2009.

[25] Z. Sun and S. Wang, "A CFD-based test method for control of indoor environment and space ventilation," Building and Environment, vol. 45, no. 6, pp. 1441-1447, 2010. 


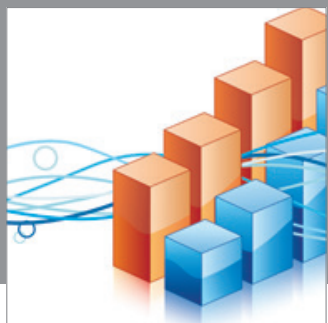

Advances in

Operations Research

mansans

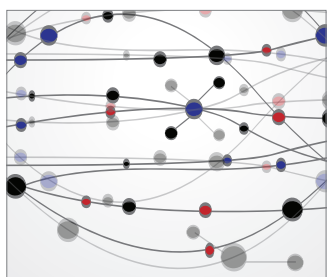

The Scientific World Journal
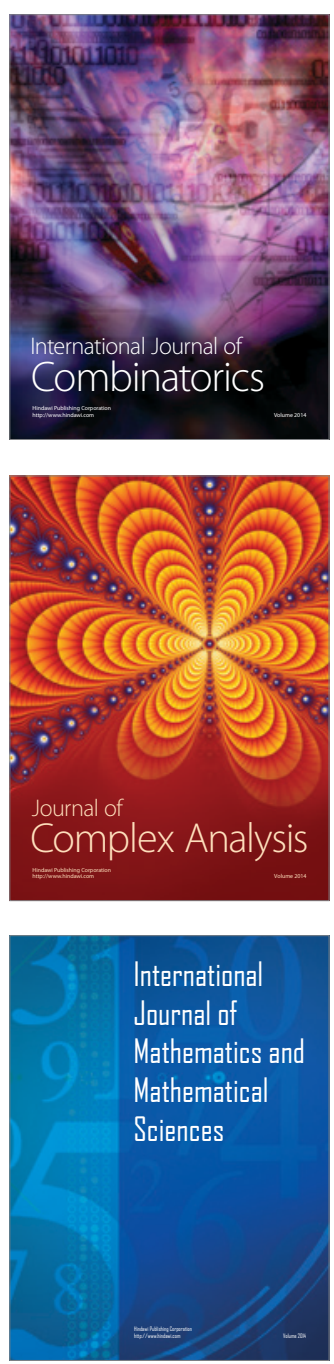
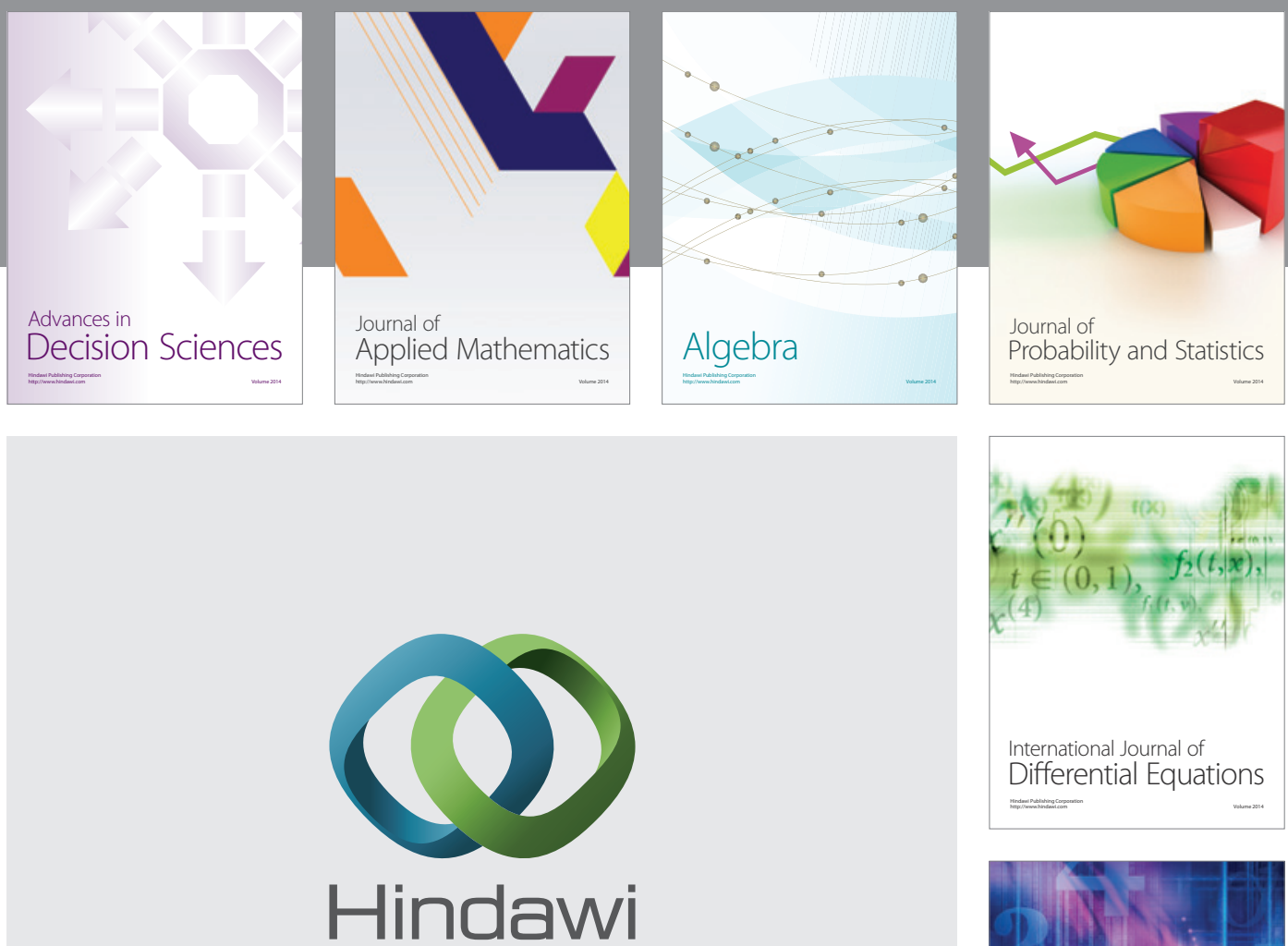

Submit your manuscripts at http://www.hindawi.com
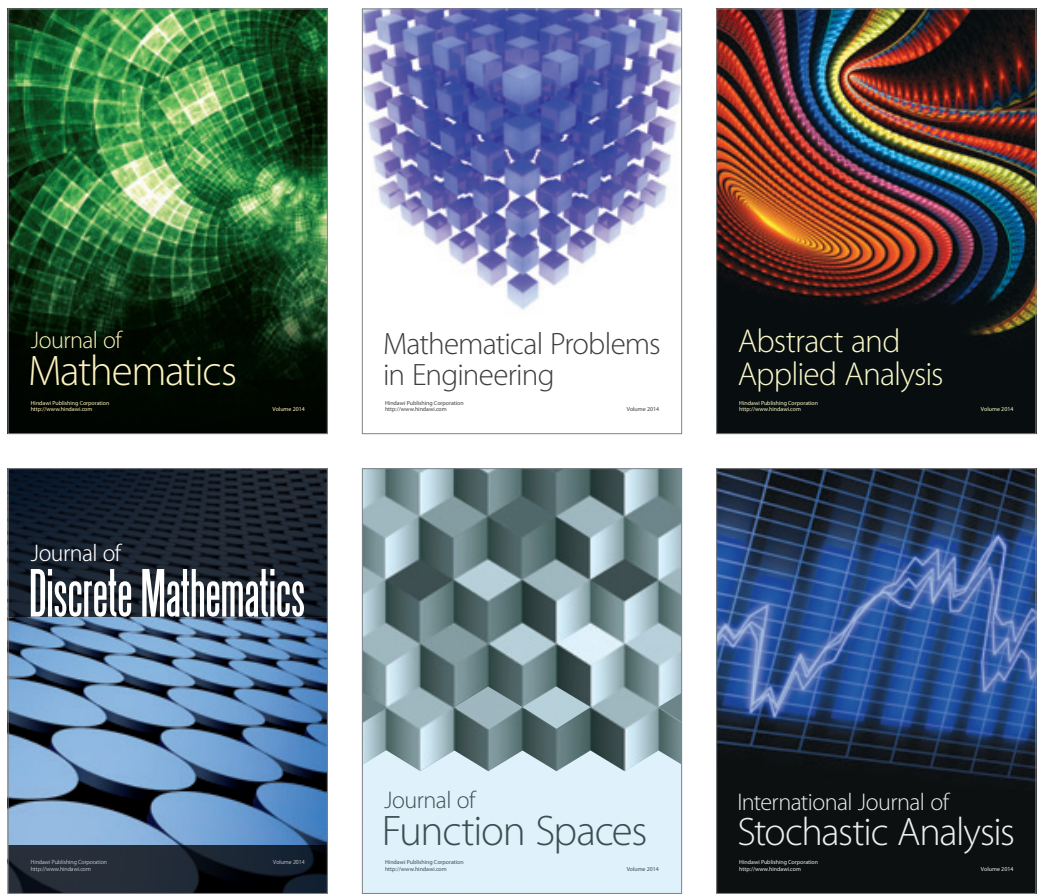

Journal of

Function Spaces

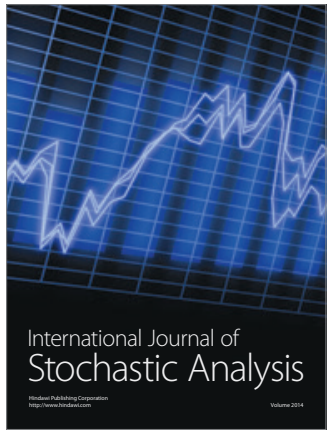

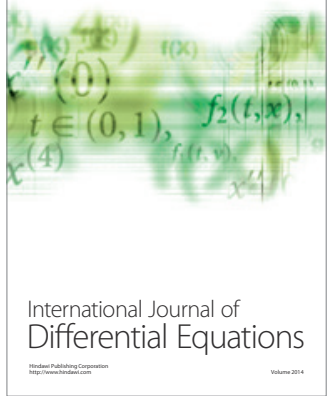
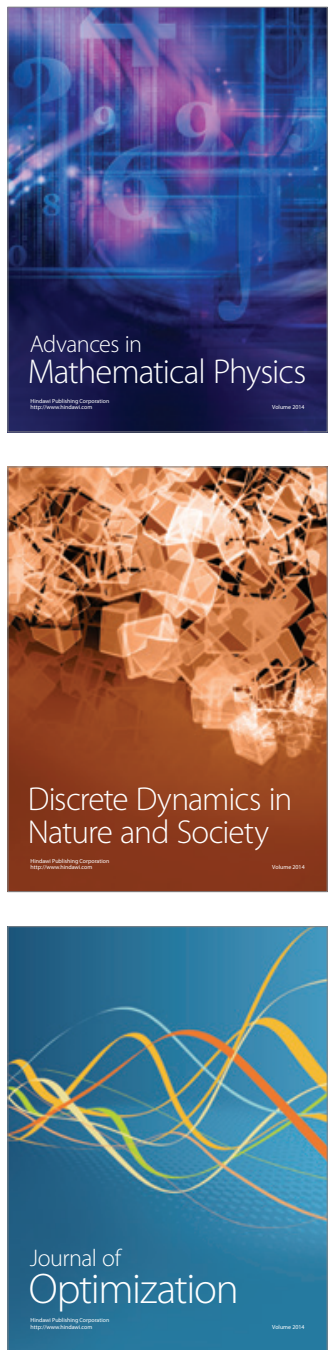